Portland State University

PDXScholar

$10-1-2011$

\title{
Field programmable gate array based reconfigurable scanning probe/optical microscope
}

\author{
Derek Brant Nowak \\ Portland State University \\ Andrew James Lawrence \\ Portland State University \\ Zechariah K. Dzegede \\ Portland State University \\ Justin C. Hiester \\ Portland State University \\ Cliff Kim \\ Portand State University
}

See next page for additional authors

Follow this and additional works at: https://pdxscholar.library.pdx.edu/phy_fac

Part of the Physics Commons

Let us know how access to this document benefits you.

\section{Citation Details}

Nowak, D. B., Lawrence, A. J., Dzegede, Z. K., Hiester, J. C., Kim, C., \& Sánchez, E. J. (2011). Field programmable gate array based reconfigurable scanning probe/optical microscope. Review Of Scientific Instruments, 82(10), 103701.

This Article is brought to you for free and open access. It has been accepted for inclusion in Physics Faculty Publications and Presentations by an authorized administrator of PDXScholar. Please contact us if we can make this document more accessible: pdxscholar@pdx.edu. 


\section{Authors}

Derek Brant Nowak, Andrew James Lawrence, Zechariah K. Dzegede, Justin C. Hiester, Cliff Kim, and Erik J. Sánchez 


\title{
Field programmable gate array based reconfigurable scanning probe/optical microscope
}

\author{
Derek B. Nowak, A. J. Lawrence, Zechariah K. Dzegede, Justin C. Hiester, Cliff Kim, \\ and Erik J. Sánchez ${ }^{\text {a) }}$ \\ Department of Physics, Portland State University, PO Box 751, Portland, Oregon 97207, USA
}

(Received 1 June 2011; accepted 6 September 2011; published online 3 October 2011)

\begin{abstract}
The increasing popularity of nanometrology and nanospectroscopy has pushed researchers to develop complex new analytical systems. This paper describes the development of a platform on which to build a microscopy tool that will allow for flexibility of customization to suit research needs. The novelty of the described system lies in its versatility of capabilities. So far, one version of this microscope has allowed for successful near-field and far-field fluorescence imaging with single molecule detection sensitivity. This system is easily adapted for reflection, polarization (Kerr magneto-optical (MO)), Raman, super-resolution techniques, and other novel scanning probe imaging and spectroscopic designs. While collecting a variety of forms of optical images, the system can simultaneously monitor topographic information of a sample with an integrated tuning fork based shear force system. The instrument has the ability to image at room temperature and atmospheric pressure or under liquid. The core of the design is a field programmable gate array (FPGA) data acquisition card and a single, low cost computer to control the microscope with analog control circuitry using off-the-shelf available components. A detailed description of electronics, mechanical requirements, and software algorithms as well as examples of some different forms of the microscope developed so far are discussed. () 2011 American Institute of Physics. [doi:10.1063/1.3643770]
\end{abstract}

\section{INTRODUCTION}

The invention of the scanning tunneling microscope ${ }^{1}$ opened the door for researchers to probe the surface of objects at the nanoscale. Further development of the probe based technique allowed for nonconductive surface imaging using atomic force microscopy (AFM) ${ }^{2}$ A customization of the AFM replaced the laser feedback system of maintaining sample-probe distance with a quartz tuning fork, resulting in a shear force microscopy (SFM) ${ }^{3}$ method. Due to the high Q-factor (sensitivity) and small footprint, tuning fork oscillators have found applications in many forms of microscopy, including magnetic force, ${ }^{4,5}$ ultrasonic, ${ }^{6}$ low-temperature, ${ }^{7}$ and in situ liquid ${ }^{8}$ imaging. The introduction of tuning fork based feedback scanning force microscopes has allowed for an increased sensitivity over previously used bimorph lateral scanning for near-field microscopes ${ }^{9-11}$ and has thus reduced optical resolutions to tens of nanometers. We employ the tuning fork oscillator method in our design due to the high degree of flexibility and sensitivity.

Traditional optical imaging suffers from diffraction which, according to Abbe's theory, limits the spatial resolution of traditional light microscopes to $\sim \lambda / 2$. With the use of pinhole apertures, a confocal technique can be used to improve the resolution to $\sim \lambda / 3$. A comparable increase in resolution is also obtainable with two-photon excitation (TPE) methods. ${ }^{12}$ Advances in far-field super resolution fluorescence methods have further reduced optical resolutions. ${ }^{13-17}$ In addition to fluorescence, polarization information can be analyzed to determine index of refraction differences and

a) Author to whom correspondence should be addressed. Electronic mail: esanchez@pdx.edu. magnetic Kerr polarization rotation in magnetic samples imaged in reflection mode. ${ }^{18,19}$ The microscope design allows for all of these methods with the use of the far-field components described herein.

Ultra-high near-field resolutions can be obtained by integrating the scanning probe and far-field optical components. A more complex form of the microscope design presented here demonstrates apertureless near-field microscopy using an epi-illumination mode of probe tip excitation. ${ }^{20-23}$ The acronym TENOM (tip enhanced near-field optical microscopy) has been assigned to this type of near-field imaging. ${ }^{24}$ The imaging probes are electrochemically etched and then tailored using a focused ion beam ${ }^{25,26}$ to create geometries that are optimal for high field enhancement with TPE molecular fluorescence, tip enhanced Raman scattering and other forms of nonlinear imaging. ${ }^{22,27-29}$

The development of the described system was motivated by the need for an optical/topographic imaging system with more versatility in both software and hardware than has typically been available. Most commercial systems tend to be black boxes that can be modified with some limited scripting. The system described here has high photon sensitivity and is completely configurable, both in hardware and software, with simple off-the-shelf adaptability and LabVIEW programming. Additionally, this system performs far-field and near-field techniques with single molecule detection capabilities and is affordable by most laboratories.

\section{SFM ELECTRONICS}

Since the near-field configuration sets the highest demands both on the optical and scanning requirements of the 


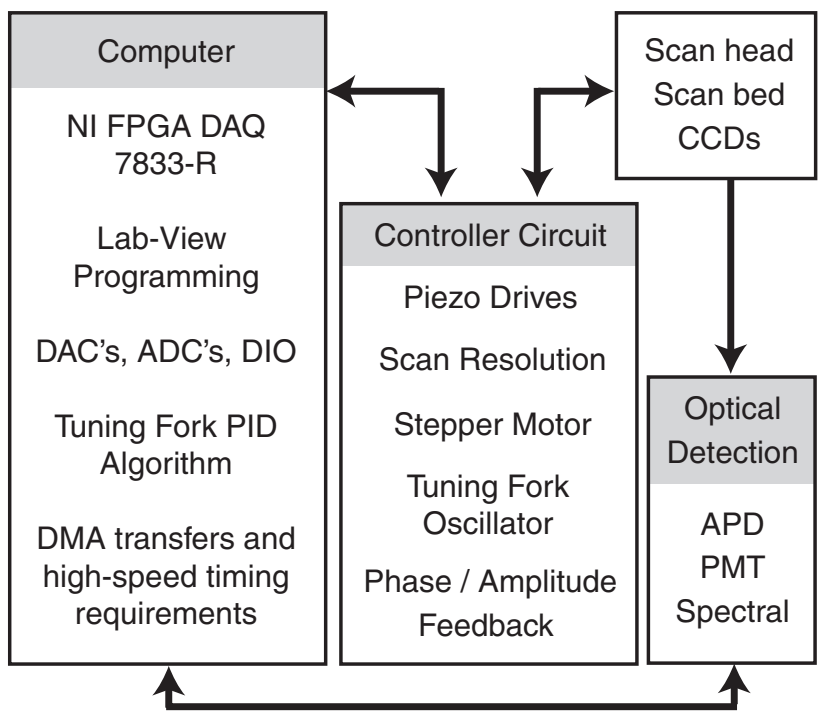

FIG. 1. Overview connection diagram of the microscope system based on a single computer and FPGA DAQ card. The FPGA interfaces with a custom controller circuit, which conditions the inputs and outputs. Signals from the controller circuit connect directly to the microscope. The microscope detection systems send data back to the computer via the FPGA and/or high-speed serial ( aaxi $^{\mathrm{TM}}$ ) cards (for interfacing with cameras).

design, the spatial XY movement and controls must allow for nanometer to sub-nanometer precision. Due to the small interaction volume ( $\sim 30 \mathrm{~nm}$ diameter) of the localized imaging field, the $\mathrm{Z}$ motion of the probe must also be carefully controlled within only a few nanometers of the surface. This level is necessary in order to properly illuminate and scan the sample without tip or sample damage. The mounted tuning fork imaging probe is set within a few nanometers above the sample surface through constant height feedback scanning, while maintaining alignment within the diffraction limited illumination spot.

The core of the SFM is a low cost data acquisition card (NI 7833R) with an FPGA processor (Xilinx), integrated digital to analog converters (DACs) and analog to digital converters (ADCs), digital I/Os (DIO), and direct memory access (DMA) transfer capabilities, outlined in Figure 1. The 7833R DAQ card has $3 \times 10^{6}$ programmable logic gates, 8 ADCs with bandwidths of $200 \mathrm{kHz}, 8$ DACs with bandwidths of 1 $\mathrm{MHz}$, and 96 DIOs at $40 \mathrm{MHz}$. The FPGA is operated at 40 $\mathrm{MHz}$, giving digital timing resolutions and instruction clock cycles at $25 \mathrm{~ns}$. The parallel nature of the FPGA allows all of the analog and DIO ports to be accessed simultaneously.

The analog requirements for the detection of phase and amplitude from the AFM tuning fork, along with the high voltage requirements for the positioning piezos, are designed on printed circuit boards (PCBs). The controller circuit boards are designed to be modular, outlined in Figure 2, allowing for easy future design revisions and upgrades. All of the PCBs were developed in the freely available software package PCB123 (v2 and v4), developed by Sunstone Circuits (Mulino, OR). Careful design was necessary to isolate high speed digital communication from analog voltages, including power supply filtering, ferrite beads, ground planes and paths, and routing of signals on the circuit board..$^{30,31}$ All

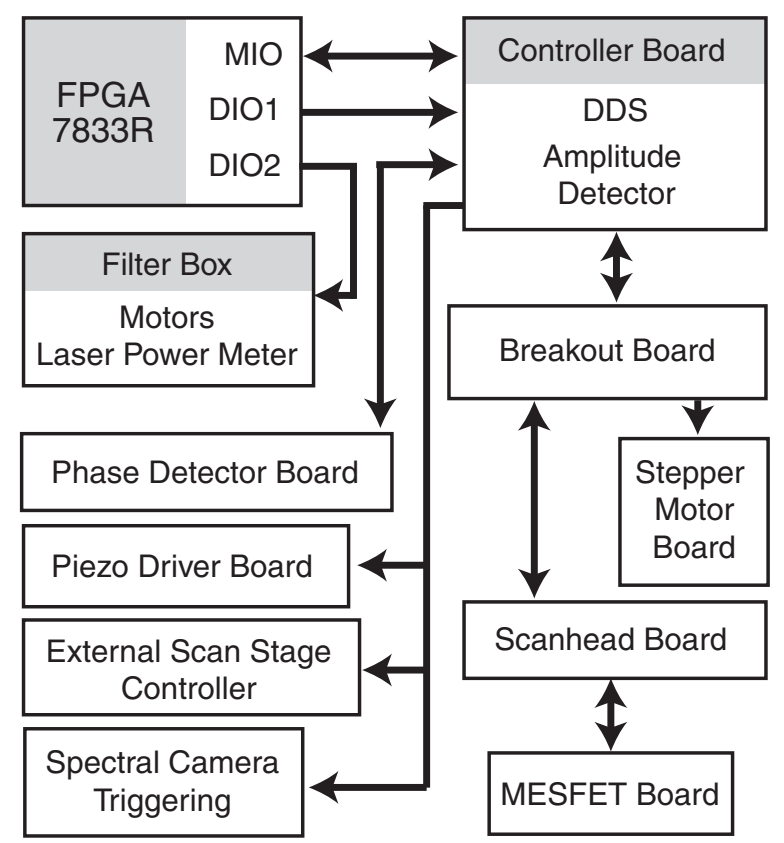

FIG. 2. A block diagram of the analog circuitry layout on multiple PCBs to allow for modularity in design. The arrows indicate the direction of data flow through the controller.

PCBs are of four layers, providing flexibility in routing so that one plane is reserved as a solid ground plane, with a common star ground on the main power supply. The digital and analog components are isolated from one another whenever possible by separating the ground layers.

Piezos are driven with high voltage (HV) operational amplifiers (PA88) from APEX Microtechnology (Cirrus Logic). ${ }^{32}$ The high voltage op-amps are configured in a bridge circuit using a gain amplifier and a unity gain inverter. In this arrangement, the output voltage swing is twice that of a single amplifier, and the slew rate is also doubled. In the bridge configuration, the PA88 differential voltage output is $600 \mathrm{~V}$ with a slew rate of $4 \mathrm{~V} / \mathrm{ms}^{33}$ The microscope design can independently control five piezos, simultaneously.

A high-resolution versatile scan algorithm is used to drive the PA88s or third-party piezo control systems (Figure 3). Two DACs are used: one that applies a static voltage offset, and one that applies the raster scanning voltage. These are summed together using an OP471 op-amp. The scanning output is sent to a programmable voltage divider (Analog Devices AD5290) to adjust the scan size while maintaining resolution. The AD5290 IC has 256 possible divisions of resistance, lowering the theoretical scanning resolution to $6 \mathrm{pm}$ per DAC division.

The tuning fork is electrically driven ${ }^{34,35}$ with a direct digital synthesizer (DDS) oscillator at or near resonance. The DDS (AD9835) allows for precise frequency control within $0.01 \mathrm{~Hz}$. A MESFET (NXP-BF992) configured as a source follower preamplifier is placed in close proximity to the tuning fork with a gain of $\sim 10^{6}$. The signal is further amplified with two operational amplifiers (AD8672) for a combined gain of $10^{3}$. The signal is split to a rms detector (AD637) to detect amplitude ${ }^{36}$ and a high-speed comparator circuit to determine the phase in relation to a reference from the DDS. 


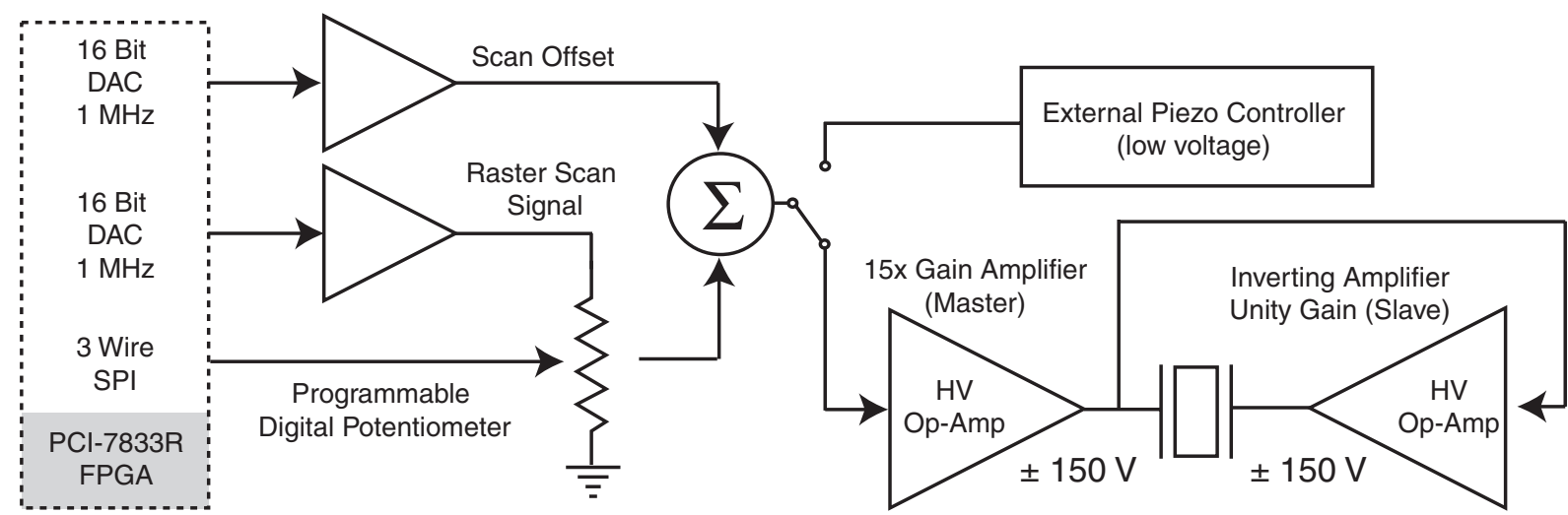

FIG. 3. High-resolution scan circuitry with piezo driver bridge circuit. The piezo actuator is isolated from a ground reference voltage, providing it with a full voltage swing of $600 \mathrm{~V}_{\mathrm{pp}}$. Flexibility in design allows for easy integration of external third-party piezo drivers.

The phase detector is used as the feedback signal for the proportional integral differential (PID), creating a phase lock loop. ${ }^{37-40}$ The slow response of the amplitude is used only for resonance tuning. Figure 4 shows the logic of the phase detection circuit, based on a high-speed comparator (Linear Technologies LT1712) compensated for hysteresis, combined with a four-quadrant analog multiplier (AD633) and a fourthorder low pass filter (LTC1563). Figure 5 shows the output of the amplitude and phase measurements. One must take care not to implement phase demodulation chips with $\mathrm{GHz}$ capable ranges as prototyping with this device demonstrated noise complications from interference with cellular phone frequencies and will directly affect the feedback signal.

A stepper motor with a modular gearbox is used as a coarse approach guide to place the tip onto the sample surface. The motor is run with a quarter step routine using programmed logic in the FPGA with four DIO ports, optocoupled (Vishay TCMT4100) to TIP31 high current transistors. The FPGA controls the approach by extending the Z-piezo until the surface location is determined.

\section{MECHANICAL DESIGN}

The design of the optical pathways for the microscope system is based on a custom inverted optical microscope. The design allows for the incorporation of an SFM scan head while maintaining a simplified optical system. Due to the sensitivity of SFM imaging, the height of the microscope is kept to a minimum. The system base parts were manufactured from cast iron, stainless steel, and aluminum. The main reason for this choice in materials is ease of manufacture, allowing researchers to machine the parts themselves. This material selection is not the optimum for the SPM requirements, but with careful machining and design, it is possible to place the sensitive locations of the microscope in the center of most parts. This allows for the radial expansion and contraction to be minimized at those locations. ${ }^{41-43}$

The microscope was carefully designed so that the custom machined components would fit compatibly with thirdparty parts while maintaining simple dimensions. Figure 6 shows all the major parts of the microscope. The inverted

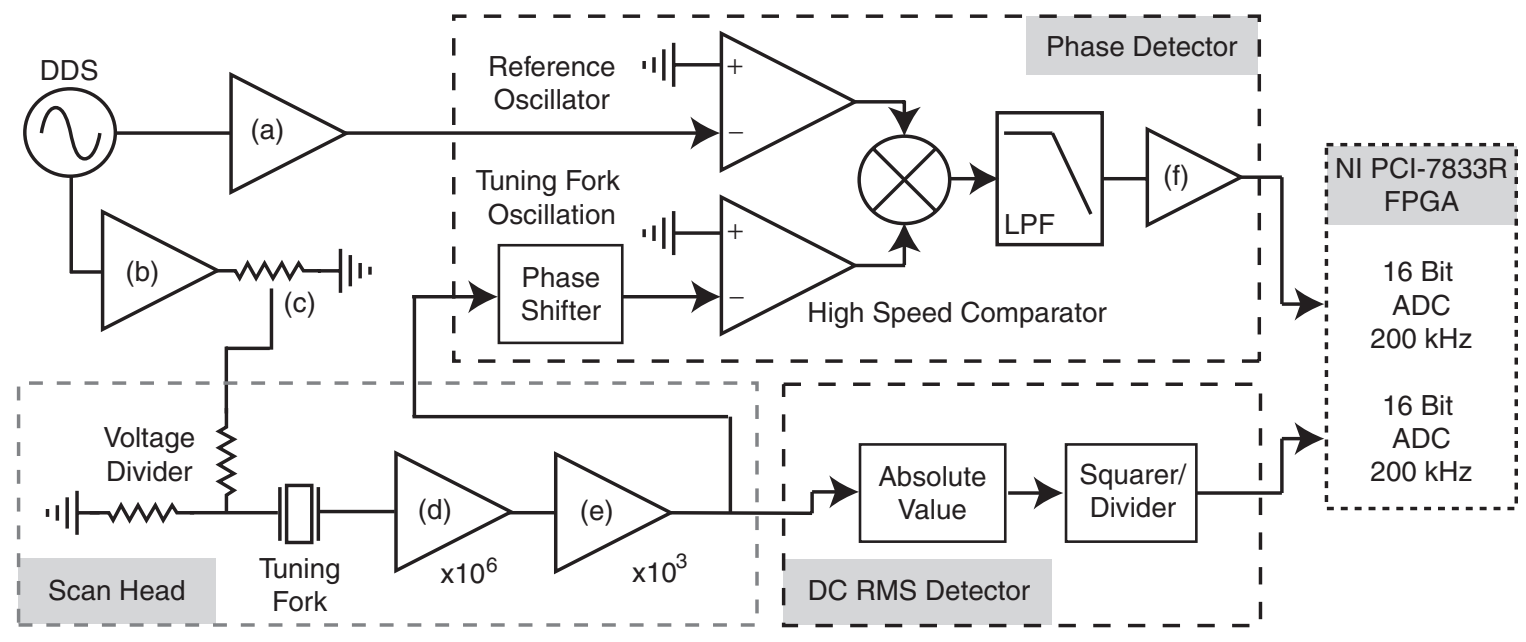

FIG. 4. Tuning fork oscillation and detection scheme: The reference oscillator is compared to the return oscillation from the tuning fork. The tuning fork oscillation can be phase-shifted to correct for any stray system capacitance. The tuning fork oscillation amplitude is monitored to aid in tuning of the oscillator, thereby determining the quality factor of the system. (a) The summing setpoint amplifier conditions the reference signal ( $\pm 5 \mathrm{~V}_{\max }$ ). (b) The amplifier conditions the drive signal for the long signal pathway to the scan head, where the signal is then voltage-divided for a range of $0.3-7.0 \mathrm{mV}$ near the tuning fork. (c) The digital potentiometer (3-wire SPI) controls the drive voltage. (d) MESFET preamplifier. (e) The two-stage amplifier acts as a band-pass filtering between 11 and $73 \mathrm{kHz}$, (f) which conditions the phase output for the proper dynamic range of the ADC. Details for phase detector outlined in text. 


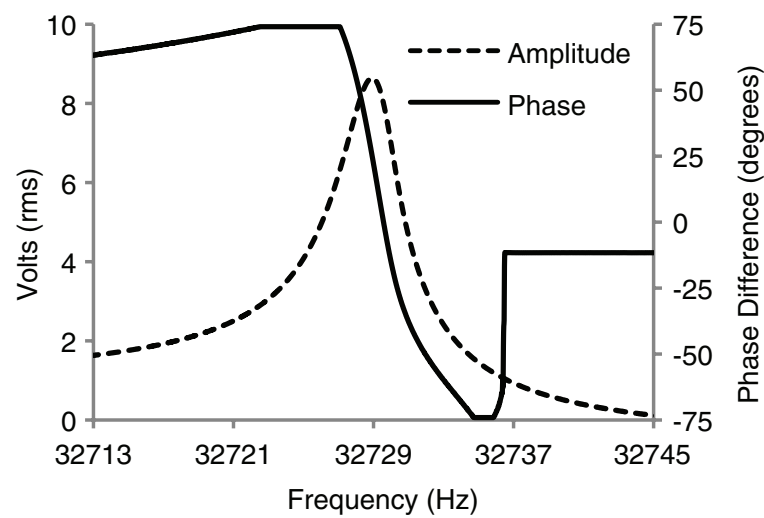

FIG. 5. Graph of the amplitude and phase relationship for the quartz tuning fork recorded by the feedback electronics. The saturation of the phase at $\pm 75^{\circ}$ is an artifact of the phase detector in using the full dynamic range of the ADC. The phase region after $32737 \mathrm{~Hz}$ is an artifact of the return signal (outside of resonance region) being too low for comparison. The amplitude demonstrates a quality factor of $\sim 7000$ for a bare tuning fork in ambient conditions, with asymmetry due to the parasitic capacitance of the tuning fork.

microscope base is built on an optical breadboard (Thorlabs MB1218) and is supported by four stainless steel posts (Thorlabs P8). These posts support the custom baseplate, backplane, and X Y piezo scan bed (Physik Instrumente P733.2CL). An X Y Z scan bed would be advantageous, as the $\mathrm{Z}$ translation could be used to help maintain proper vertical focus (not used in the presented design). The excitation laser is guided to a dichroic filter by a periscope which consists of two mirrors at $45^{\circ}$ to the laser incidence and orthogonal to each other, one mounted to the breadboard and one to the underside of the baseplate (Thorlabs KM100, MA452, PF10-03-P01; Melles Griot 07 TXS 223). The excitation and collection optics are mounted under the baseplate using Thorlabs $30 \mathrm{~mm}$ cage system parts. The objective lens is focused with a $\mathrm{Z}$ translator (Thorlabs SM1Z), followed by a cube to hold a dichroic filter (Thorlabs C4W, B1C, B4C,
B5C). The collection pathway continues down to a $45^{\circ}$ steering mirror (Thorlabs KCB1, PF10-03-P01) that redirects the light to an 8:92 beam splitter (Thorlabs CM1-BP108), where $92 \%$ of the light is passed through emission filters to be fiber coupled (Thorlabs K6X, F810SMA-543). The remaining $8 \%$ is focused with a tube lens (Thorlabs AC254-200-A) and allowed to pass through a 50:50 beam splitter (Thorlabs CM1-BS013). One path leads to a magnifying lens (Thorlabs AC254-40-A) to a CCD camera (KPC-S500B) to view the sample surface. The other path has a Bertrand lens system (Thorlabs AC254-50-A, A375TM-A) to view the bottom of the scan head. All of the optical pathways are enclosed in tubes (Thorlabs SM1L30, SM1L10, SM05L10) to minimize stray light. The optical pathways are optimized for infinity corrected objective lens but could be rearranged for older style objective lenses. ${ }^{44}$

The custom scan head is held above the objective lens with a custom backplane and a translation stage (Thorlabs LNR50). The vertical movement of the translation stage is controlled by a stepper motor (Oriental Motor PK243B1ASG36) mounted to a lead screw (Universal Precision Lead Screw and Side Arm Cartridge Assembly, 1/2 in. diameter, 1/4 in. lead, 2 in. travel). The scan head design includes a low power, adjustable laser pointer, a steering mirror, a tuning fork preamplifier circuit board, and a miniature CCD camera, shown in Figure 6. The piezo stack (Newport MFM-075 Flexure Stage; $\mathrm{X}-\mathrm{Y}$ segmented and $\mathrm{Z}$ tube piezos - Boston Piezo Optics) is placed in the center of the aluminum scan head to minimize the effects of scan drift due to thermal expansion of the aluminum. The laser pointer (Thorlabs CPS198) and CCD (KPC-S500B) are used to align the probe into the focused laser spot from the objective lens in the inverted microscope, shown in Figure 6. It should be noted that the laser pointer was originally intended to illuminate the end of the probe; when the probe was near the diffraction limited spot, the diffraction pattern for the tip would give an approximation of the location of the end tip of the probe. It was
(1)

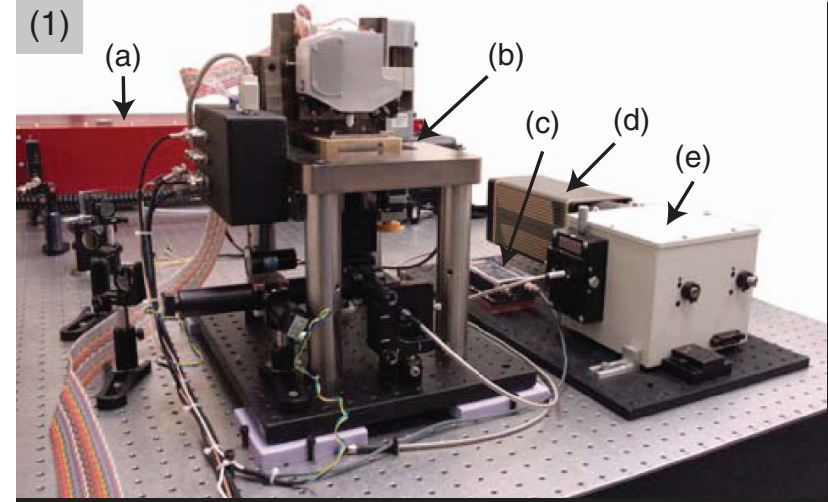

(1) Microscope System

(a) Ti:Sapphire Laser

(b) Microscope

(c) APD

(d) ICCD

(e) Monochromator

\begin{abstract}
(2) Microscope
\end{abstract}
(f) Backplane

(g) Electronics Box

(h) CCDs

(i) Lead Screw

(j) Scanhead (k) Scan Bed

(l) Base Plate

(m) Stepper Motor

(n) Dichroic Beam Spitter

(o) Fiber Coupler

(p) Support Posts

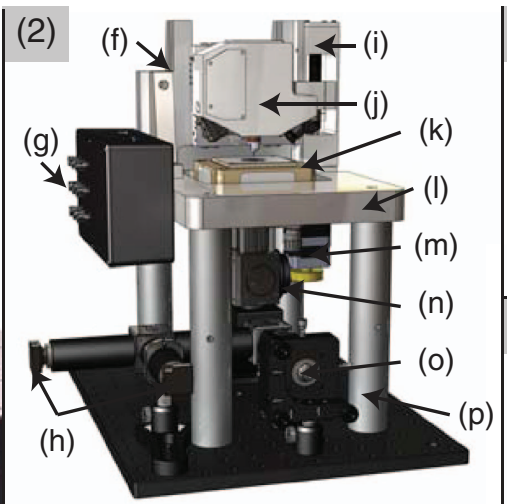

(3) SFM Scan head

(q) Electronics Cover

(r) Mirror

(s) CCD

(t) Tuning Fork

(u) Preamplifier

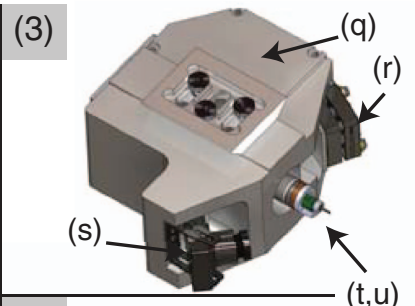

(4) (v)

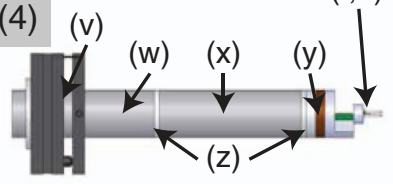

(4) Piezo Stack

(v) Flexure Stage

(w) XY-Piezo

(x) Z-Piezo

(y) Plastic Spacer

(z) MACOR

FIG. 6. (Color online) (a-z) The major elements for the microscope system. (1) Photograph of the overall physical system with support lasers and optical detection systems. Images 2-4 are detailed SolidWorks renderings of the microscope (2), scan head (3), and piezo stack (4). Further details on parts outlined in text. 
discovered that side illumination of the tip with multiple LEDs would provide adequate shadowing of the tip when viewed from below. The current design has two LEDs mounted on the CCD camera, and could be modified to integrate an AFM cantilever optical feedback system on the microscope, which would require laser and mirror components and the addition of a quadrant photodiode.

\section{SOFTWARE ALGORITHMS}

The computer automation of the microscope system has two independent layers of computer processing. The FPGA comprises the first layer, running all the core logic and automated routines that require autonomy from the Windows OS: oscillator tuning, data acquisition scan, approach, and the PID algorithm. The second layer is the user interface that runs the graphical user interface (GUI) that interacts with the FPGA program to control the microscope. The second layer requests information from the FPGA to display and save incoming data, allowing the user to change parameters in real-time in a Windows operating system environment, outlined in Figure 7. The programming environments used for the development of the microscope are National Instruments LabVIEW versions 8.2 to 2010 .

The FPGA runs a digital proportional-integraldifferential feedback loop that controls the $\mathrm{Z}$ piezo. National Instruments provides an FPGA PID control toolkit that includes a discrete PID algorithm. A slight modification to the PID algorithm provided functionality at the bandwidths needed for scanning probe microscopy. The initial difficulty with the NI PID algorithm was a lack of dynamic range in the integral term, causing this term to dominate at even the smallest of values. This made it difficult for the system to maintain surface displacement over areas with small $(<10 \mathrm{~nm})$ topographic features, such as mica. A change to the internal code reduced the responsiveness of the integral term, ${ }^{45}$ thereby allowing for successful scans.

Another vital algorithm in the FPGA logic is the scan algorithm. The scan algorithm requires the synchronization of

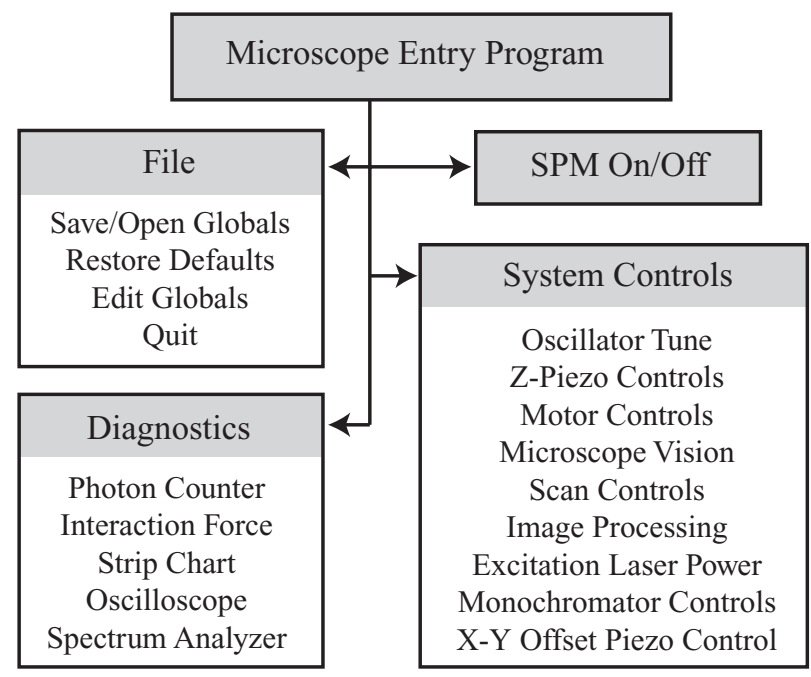

FIG. 7. Basic GUI structure for the software of the microscope system.

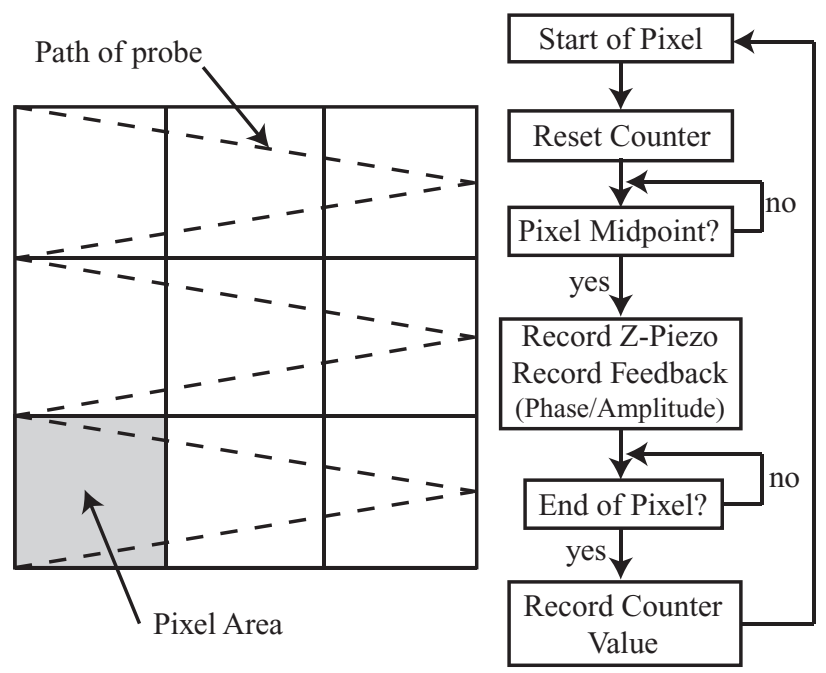

FIG. 8. Scan algorithm for a $3 \times 3$ pixel scan area. The dashed line represents the path of the probe as it creates a trace and re-trace line for each y-axis pixel. The algorithm for data synchronization runs in its entirety for each pixel.

multiple sub-routines that are interacting with each other. The highest level of the algorithm is the raster scan generator. The raster scan creates a trace and re-trace line in the $\mathrm{X}$ axis for each pixel depth in the $\mathrm{Y}$ axis. Figure 8 shows a dashed line that represents the raster scan path of the probe. The raster scan is composed of two triangle waveforms that are generated in real-time as the probe scans. A linear interpolation function is used in conjunction with Boolean flags to determine if the scan is in a trace, re-trace, up, or down parts of the waveforms. The $\mathrm{X}$ axis and $\mathrm{Y}$ axis are generated from the same algorithm, with the $\mathrm{Y}$ axis function slowed to create a longer period than that of the $\mathrm{X}$ axis. The two scanning waveforms are synchronized to start simultaneously by the FPGA.

The data collection during the scan is handled by three routines that are continuously checking for the start, midpoint, and end of pixel conditions, while calculating the new values for the next pixel. The start pixel routine and the midpoint pixel routine check every clock cycle of the FPGA in a single cycle timed loop for the value that defines the start pixel or midpoint pixel from the linear interpolation waveform function in real time for the $\mathrm{X}$ axis. Since the scan waveforms can only update once per clock cycle, this guarantees that values in the waveform will not be missed. The start pixel routine will also handle the DMA transfer for the pixel information, photon counts, topographic, feedback error, current $\mathrm{Y}$ line, and $\mathrm{X}$ pixel. Based on the current direction of the microscope (trace, re-trace) and the number of imaging pixels, the FPGA will calculate the next pixel parameters (start, stop, and midpoint) in real-time, based on the current location of the imaging probe in the scan waveform algorithm. Any Windows request to move the tip to a new location (i.e., top of page, bottom of page, start and stop scanning) will be interpolated to the new location in single DAQ step increments, at the imaging scan rate defined by the user.

The approach algorithm is designed to ensure that the tips are not damaged during approach. During the extension of the piezo, the feedback signal is closely monitored for a change (signaling the location of the surface). If a change occurs, the 
approach algorithm is stopped and the control of the $\mathrm{Z}$ piezo is passed to the PID algorithm. If the surface is not located, then the piezo is retracted and the stepper motor is instructed to take a step toward the surface. A delay is used after the step instruction to allow for motor settling time. This process is repeated until the surface is located. During run-time, the rates for piezo extension and retraction and stepper motor delay time and number of steps are changeable by the user.

\section{TOPOGRAPHIC AND OPTICAL IMAGING}

The versatility of the system makes it useful as an SFM, a confocal scanning optical microscope, or a near-field system. The presented system design utilizes an external closed loop $\left(100 \times 100 \mu \mathrm{m}^{2}\right)$ scan bed (Physik Instrumente P733.2CL), in order to achieve very high precision and accuracy in positioning. The ability of the microscope to delicately scan a sample in a non-contact shear force mode without damaging the tip or sample is demonstrated by the sharp tungsten tip in Figure 9 that shows a closed-loop scanned topographic and phase feedback image of platinum coated silicon pits (calibration grid) with a $1.0 \times 1.0 \mu \mathrm{m}^{2}$ periodicity and a scan rate of $0.3 \mathrm{~Hz}$. The ability to scan the sample allows for optical imaging since an objective is present under the sample and designed primarily for epi-illumination and collection, thereby allowing for the optical scanning in many different modes with high precision.

The optical setup of the microscope can be adapted to accommodate many forms of optical imaging, including basic reflection, transmission, fluorescence, Kerr, Raman, or other modes. The point-scanning reflection mode functionality of the microscope is demonstrated in Figure 10, which shows a magnetic glass sample (Metglas 2065SA1). The image was taken at a scan speed of $1.0 \mathrm{~Hz}$ using $488 \mathrm{~nm}$ at $110 \mu \mathrm{W}$ illumination from an argon ion laser. The difference in $\mathrm{Z}$ height can be seen in the apparent depth of field on the image. This image was taken with a high light level avalanche photodiode (APD) (Thorlabs APD110A), as opposed to a high sensitivity single photon level APD. Although the APD allowed for very low light level imaging, a photomultiplier tube or even sili-

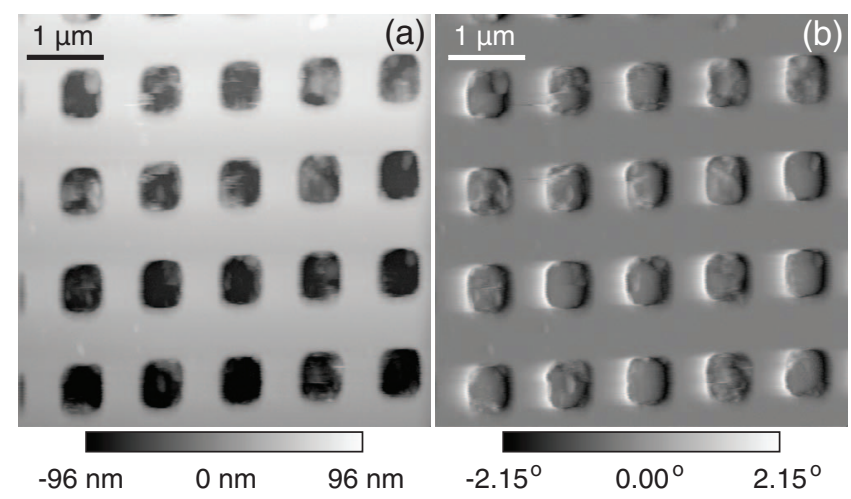

FIG. 9. Shear force images of a calibration grid (Digital Instruments, Veeco, PG platinum coated calibration grid $1.0 \times 1.0 \mu \mathrm{m}^{2}$ periodicity). (a) The topographic image in the trace direction (left to right). (b) The error in the phase feedback signal in the re-trace direction (right to left). Both images were acquired at a scan rate of $0.3 \mathrm{~Hz}$.

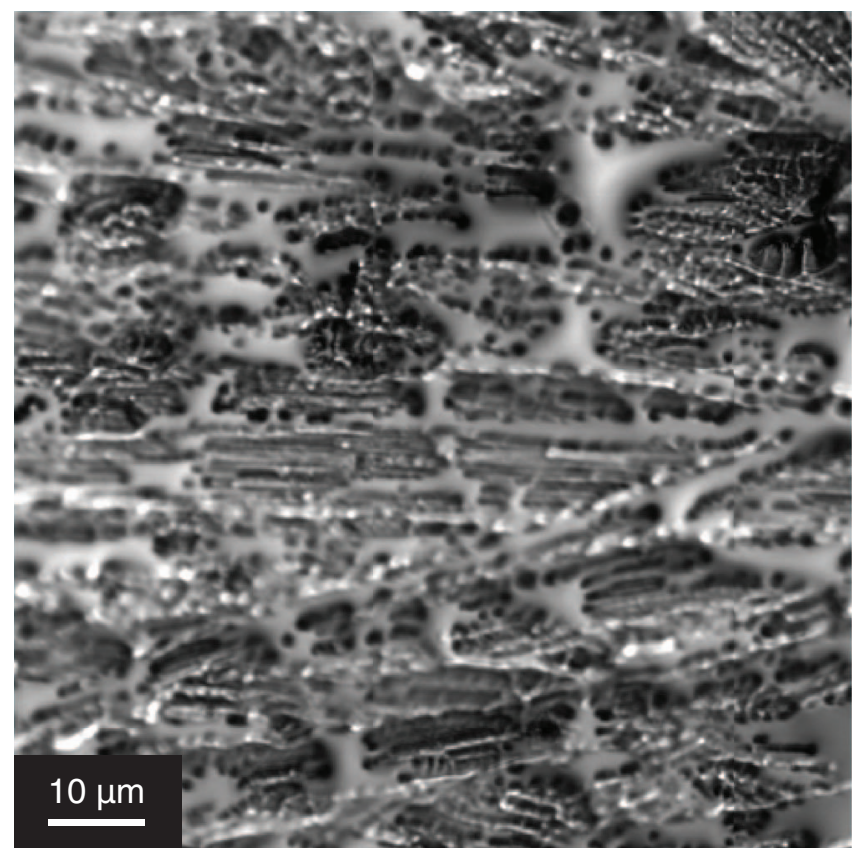

FIG. 10. A reflection optical image of magnetic glass, Metglas 2065SA1. The image was acquired at a $1.0 \mathrm{~Hz}$ scan speed using $488 \mathrm{~nm}$ laser illumination at $110 \mu \mathrm{W}$ (image size $96.0 \times 96.0 \mu \mathrm{m}^{2}$ ).

con photodiode would suffice. The physical arrangement was similar to the Kerr optical setup as described below, with the use of just one APD.

The flexibility of the instrument is further demonstrated by adaptation to a polarization based imaging mode using the magneto-optic Kerr effect. Figure 11 outlines the optical pathway employed for Kerr imaging. A beamsplitter (Thorlabs CM1-PBS251) separates the reflected light into its $s$ and $p$ components, which are collected separately by APDs (Thorlabs APD110A). The polarization of the incident light is set such that, in the absence of Kerr effect induced rotation, the $s$ and $p$ channels are equal in intensity. The difference signal between $s$ and $p$ is the result of the Kerr rotation and an indication of the level and direction of magnetization of the surface. This method was used to image (Objective Nikon M-Plan 100 \#323887) a magneto-optic data disk, as seen in

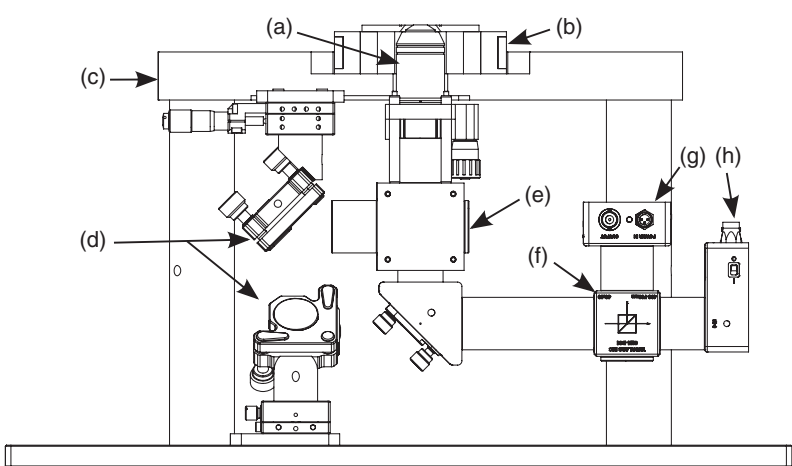

FIG. 11. Diagram of the polarization microscope mode. System includes polarization optics to differentiate both $s$ and $p$ orientations to elicit Kerr optical rotation of the sample; (a) objective lens, (b) scan bed, (c) base plate, (d) periscope, (e) beamsplitter, (f) polarizing beamsplitter, (g) APD for the s channel, (h) APD for the $p$ channel. 

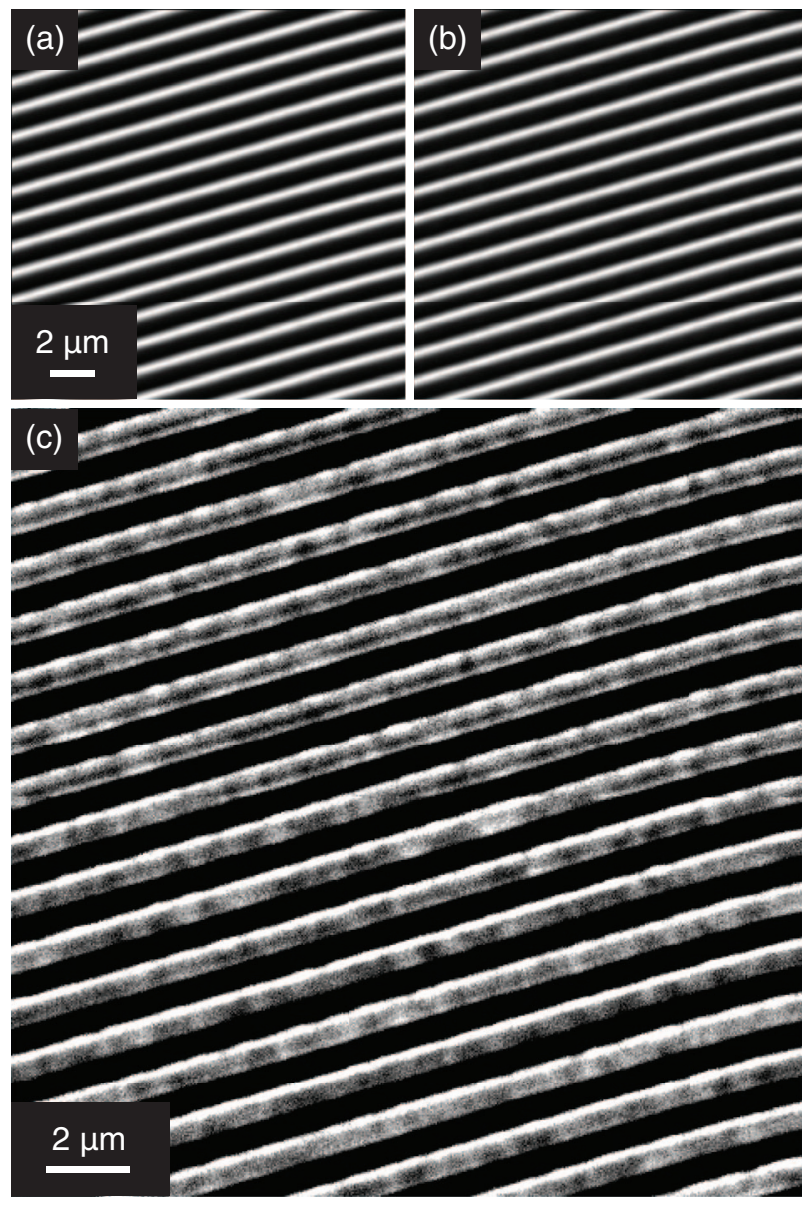

FIG. 12. Far-field Kerr image of a magneto-optical disk obtained using a difference method revealing individual magnetic bits. Image (c) is the difference signal between the $s$ channel (a) and the $p$ channel (b). Image was acquired with a $2 \mathrm{~Hz}$ scan speed using $488 \mathrm{~nm}$ laser illumination at $110 \mu \mathrm{W}$ (image size is $21.0 \times 21.0 \mu \mathrm{m}^{2}$ ).

Figure 12. In Figures 12(a) and 12(b), the images show strips of reflected light from the different sectors on the magnetooptical (MO) disk, indicating the $s$ and $p$ polarizations respectively. No information is easily discerned from these images; however, Figure 12(c) shows the difference in the $s$ and $p$ channel, thus indicating the magnetic bit structure of the MO disk. The images were acquired at a $2.0 \mathrm{~Hz}$ scan speed using $488 \mathrm{~nm}$ laser illumination at $110 \mu \mathrm{W}$.

To demonstrate the high spatial optical resolution of the microscope in one configuration, near-field multi-photon imaging was performed. A sample was prepared with a solution of Rhodamine $6 \mathrm{G}$ molecules in methanol at a $1.0 \mu \mathrm{M}$ concentration pipetted onto a coverglass slip (Gold Seal No. $1,25.0 \times 25.0 \mathrm{~mm}^{2}$ ) and allowed to air dry for several minutes. The resulting sample demonstrated dye clusters on the order of tens to hundres of $\mathrm{nm}$. Figure 13 compares the optical far-field imaging to the optical near-field and the topographic profile of the sample. The excitation wavelength was held constant at $833 \mathrm{~nm}$ with a mode-locked Ti:Sapphire laser (Del Mar Photonics Trestles 50) operating at 52 fs pulses. The excitation power for the far-field imaging and near-field imaging was $27.21 \mu \mathrm{W}$ and $8.35 \mu \mathrm{W}$, respectively. The twophoton response was captured with an APD (Perkin Elmer
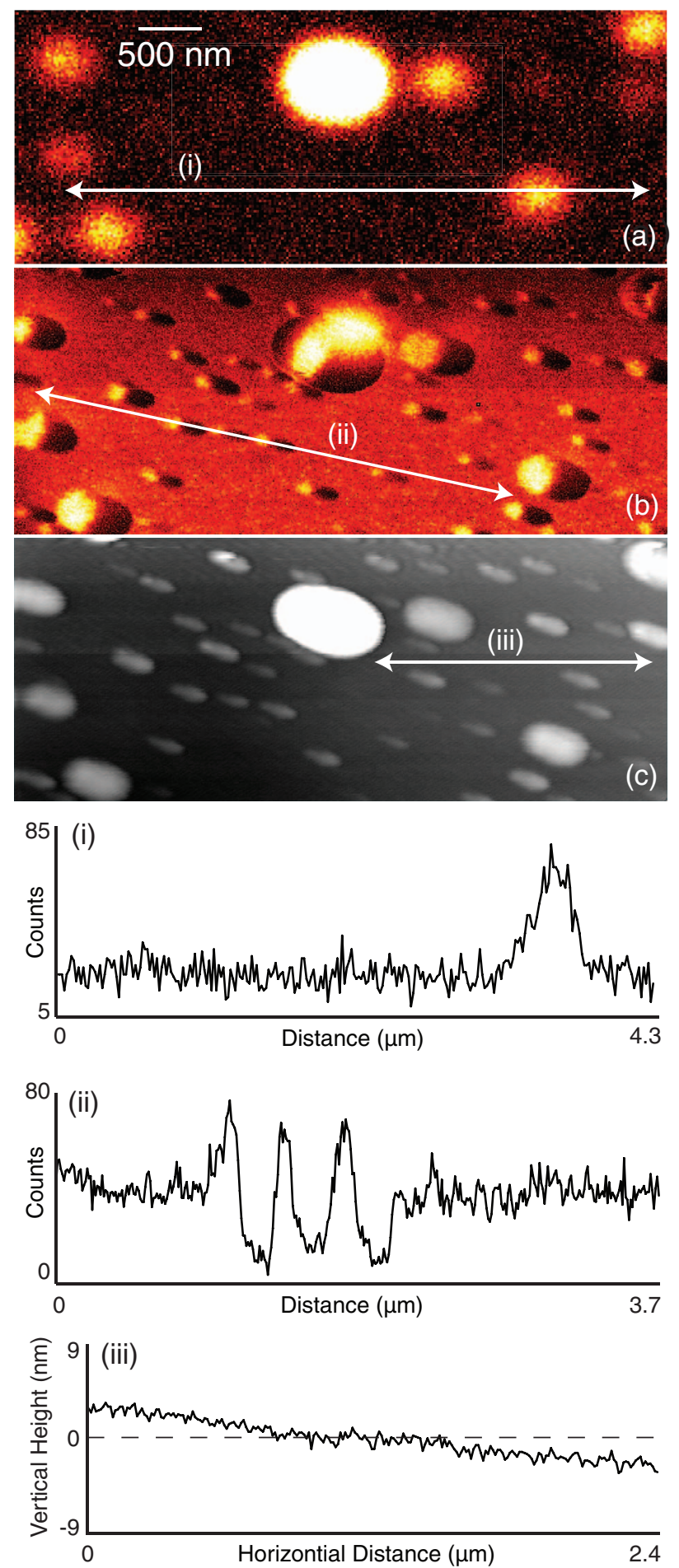

FIG. 13. (Color online) Two-photon excitation/topographic images of Rhodamine 6G dye. (a) Far-field diffraction limited image. (b) Near-field image with the same region of interest (ROI) as (a). (c) Topographic scan, simultaneously acquired with (b). Details in text.

SPQM-AQR-14-FC) using an objective lens (Olympus 1.4 NA, 60×), dichroic filter (Chroma 725dcspxr), excitation filter (RG-750), and emission filters (Chroma ET750sp-2p8). The acquisition time per image is about $5 \mathrm{~min}$, with the vertical axis represented by 512 pixels. The near-field image demonstrates the slow thermal drift of the microscope, represented by the intensity of the image fading towards the 
top. The near-field image demonstrates fluorescence quenching effects by the imaging probe; this is further demonstrated in the line analysis of the image by lowering the background level next to the optical peaks from the clusters. The custom imaging probe geometry creates the artifacts seen in the topographic image as well. A line analysis of the topographic image shows a vertical rms noise limit of the microscope at $0.6 \mathrm{~nm}$.

\section{CONCLUSION}

Continued development of the reconfigurable system with added functionality is currently under way in the author's laboratory. As a shear force microscopy tool, the addition of electric field and magnetic field imaging would add to the system's versatility. A fiber-based design and simplification of the current scan head to allow for non-transmissive samples will also be investigated for more versatile near-field imaging. Raman spectroscopy capabilities that include the integration of an intensified CCD camera with a high-resolution monochromator will allow for spectral imaging of multiple Raman lines, simultaneously. This will eliminate the need for multiple Raman notch filters, making the system more versatile. Additional implementation of the field enhancement technique will have the added benefit of amplifying the weak Raman spectral response. Ultimately, developing the microscope into a user-friendly Raman imaging system will promote the longevity of the design. Design updates are being investigated to remove the laser and mirror components from the scan head and simplify the number of cuts necessary. A future design will also include a four point illumination with LEDs to provide a cross pattern that would intersect when the tip reaches the surface. Transferring the CCD from the scan head to a holder on the base of the microscope is also being investigated.

So far, a reflection mode confocal microscope, polarization based microscope (Kerr), and tip enhanced near-field optical fluorescence microscope have been successfully completed as different forms of this microscope design. The versatility allows for high-resolution topographic imaging, single molecule sensitive far-field imaging, and high-resolution fluorescence imaging. By developing the system in a standard programming environment with commercially supported, offthe-shelf components and simplified usability, the system can be easily recreated This versatility was the original motivation for the system design, and should spark interest in the scientific community in adding technical improvements to the existing design as an open-source concept. More information about the project, relevant documentation, and software are available at our ANSOM Project website. ${ }^{46}$

\section{ACKNOWLEDGMENTS}

The authors would like to gratefully acknowledge the support of the National Science Foundation through Award No. DBI-0500812 for Biological Sciences Instrument Development. Tip fabrication was assisted through the NSF support (ECCS-NSF-0520891, with additional support from PSU, ONAMI/ONR Award Nos. (\#N00014-07-1-0457),
(\#N00014-08-1-1237), and (\#N00014-10-1-0082)), and additional personnel support for Zechariah Dzegede and Cliff Kim under the NSF DMR REU site Grant No. (NSF-069280). We are grateful to Matt Spiegelberg from the National Instruments for his FPGA expertise and the Analog Devices for help with IC designs. The authors would also like to thank Leroy Laush for circuit analysis, Marc Nisenfeld and Chuck Heino for machining assistance, and Jeff Doughty, Amber Lauer, Kelvin Pittman, and Amen Kester for their contributions to the project. Financial assistance to finalize the microscope scanning algorithms and electronic design was provided by the Western Institute for Nanoelectronics (WIN) and the Nanoelectronics Research Initiative (NRI). The authors would also like to thank Free Geek of Portland for their computational contributions to this project.

${ }^{1}$ G. Binnig, H. Rohrer, Ch. Gerber, and E. Weibel, Phys. Rev. Lett. 49, 57 (1982).

${ }^{2}$ G. Binnig, C. F. Quate, and Ch. Gerber, Phys. Rev. Lett. 56, 930 (1986).

${ }^{3}$ K. Karrai and R. D. Grober, Appl. Phys. Lett. 66, 1842 (1995).

${ }^{4}$ H. Edwards, L. Taylor, W. Duncan, and A. J. Melmed, J. Appl. Phys. 82, 980 (1997).

${ }^{5}$ M. Todorovic and S. Schultz, J. Appl. Phys. 83, 6229 (1998).

${ }^{6}$ A. La Rosa, X. Cui, J. McCollum, N. Li, and R. Nordstrom, Rev. Sci. Instrum. 76, 093707 (2005).

${ }^{7}$ J. Rychen, T. Ihn, P. Studerus, A. Herrmann, K. Ensslin, H. J. Hug, P. J. A. van Schendel, and H. J. Gäuntherodt, Appl. Surf. Sci. 157, 290 (2000).

${ }^{8}$ W. H. J. Rensen, N. F. Hulst van, and S. B. Kammer, Appl. Phys. Lett. 77, 1557 (2002)

${ }^{9}$ D. W. Pohl, W. Denk, and M. Lanz, Appl. Phys. Lett. 44, 651 (1984).

${ }^{10}$ A. Lewis, M. Isaacson, A. Harootunian, and A. Murry, Ultramicroscopy 13, 227 (1984).

${ }^{11}$ E. Betzig, J. K. Trautman, T. D. Harris, J. S. Weiner, and R. L. Kostelak, Science 251, 1468 (1991).

${ }^{12}$ W. Denk, J. H. Strickler, and W. W. Webb, Science 248, 73 (1990).

${ }^{13}$ M. Dyba and S. Hell, Phys. Rev. Lett. 88, 163901 (2002).

${ }^{14}$ K. I. Willig, S. O. Rizzoli, V. Westphal, R. Jahn, and S. W. Hell, Nature (London) 440, 935 (2006).

${ }^{15}$ M. J. Rust, M. Bates, and X. Zhuang, Nat. Methods 3, 793 (2006).

${ }^{16}$ E. Betzig, G. H. Patterson, R. Sougrat, O. W. Lindwasser, S. Elenych, J. S. Bonifacino, M. W. Davidson, J. Lippincott-Schwartz, and H. F. Hess, Science 313, 1642 (2006)

${ }^{17}$ S. T. Hess, T. P. K. Girirajan, and M. D. Mason, Biophys. J. 91, 4258 (2006).

${ }^{18}$ C. A. Fowler and E. M. Fryer, Phys. Rev. 94(1), 52 (1954).

${ }^{19}$ P. Kasiraj, R. M. Shelby, J. S. Best, and D. E. Horne, IEEE Trans. Magn. 22(5), 837(1986).

${ }^{20}$ J. Wessel, J. Opt. Soc. Am. B 2, 1538 (1985).

${ }^{21}$ Y. Inouye and S. Kawata, Opt. Lett. 19, 159(1994).

${ }^{22}$ F. Zenhausern, Y. Martin, and H. K. Wickramsinghe, Science 269, 1083 (1995).

${ }^{23}$ H. F. Hamann, A. Gallagher, and D. J. Nesbitt, Appl. Phys. Lett. 76, 1953 (2000).

${ }^{24}$ E. J. Sánchez, L. Novotny, and X. S. Xie, Phys. Rev. Lett. 82, 4014 (1999).

${ }^{25}$ M. J. Vasile, D. A. Grigg, J. E. Griffith, E. A. Fitzgerald, and P. E. Russell, Rev. Sci. Instrum. 62, 2167 (1991).

${ }^{26}$ E. J. Sánchez, J. T. Krug II, and X. S. Xie, Rev. Sci. Instrum. 73, 3901 (2002).

${ }^{27}$ T. Ichimura, N. Hayazawa, M. Hashimoto, Y. Inouye, and S. Kawata, Phys. Rev. Lett. 92, 220801 (2004).

${ }^{28}$ R. M. Stöckle, Y. D. Suh, V. Deckert, and R. Zenobi, Chem. Phys. Lett. 318, 131 (2000).

${ }^{29}$ A. Hartschuh, E. J. Sánchez, X. S. Xie, and L. Novotny, Phys. Rev. Lett. 90, 095503 (2003)

${ }^{30}$ D. Brockman and A. Williams, Analog Devices, Appl. Note AN-214.

${ }^{31}$ H. Johnson, High-Speed Digital Design (Prentice Hall, Englewood Cliffs, NJ, 1993).

${ }^{32}$ Cirrus Logic, Apex, Product Data Sheet, Rev. N, May 2009.

${ }^{33}$ Cirrus Logic, Apex, Appl. Note 44, May 2009. 
${ }^{34}$ R. D. Grober, J. Acimovic, J. Schuck, D. Hessman, P. J. Kindlemann, J. Hespanha, A. S. Morse, K. Karrai, I. Tiemann, and S. Manus, Rev. Sci. Instrum. 71, 2776 (2000)

${ }^{35}$ C. L. Jahncke, O. Brandt, K. E. Fellows, and H. D. Hallen, Rev. Sci. Instrum. 75, 2759 (2004).

${ }^{36}$ J. W. P. Hsu, A. A. McDaniel, and H. D. Hallen, Rev. Sci. Instrum. 68, 3093 (1997).

${ }^{37}$ A. G. T. Ruiter, J. A. Veerman, K. O. van der Werf, and N. F. van Hulst, Appl. Phys. Lett. 71(1), 28 (1997).

${ }^{38}$ T. R. Albrecht, P. Grutter, D. Horne, and D. Rugar, J. Appl. Phys. 69, 668 (1991).

${ }^{39}$ A. Kikukawa, S. Hosaka, Y. Honda, and R. Imura, Jpn. J. Appl. Phys., Part 1 33, 1286 (1994).
${ }^{40}$ W. A. Atia and C. C. Davis, Appl. Phys. Lett. 70(4), 406 (1997).

${ }^{41}$ B. Drake, R. Sonnenfeld, J. Schneir, P. K. Hansma, G. Slough, and R. V. Coleman, Rev. Sci. Instrum. 57, 441 (1986).

${ }^{42}$ S. Gregory and C. T. Rogers, J. Vac. Sci. Technol. A 6, 390 (1988).

${ }^{43}$ S. Park and R. C. Barrett, "Design considerations for an STM system," in Methods of Experimental Physics, edited by J. A. Stroscio and W. J. Kaiser (Academic, New York, 1993), Vol. 27, pp. 30-76.

${ }^{44}$ D. B. Nowak, A. J. Lawrence, and E. J. Sánchez, Appl. Opt. 49, 6766 (2010).

${ }^{45}$ D. B. Nowak, Ph.D. dissertation, Portland State University, 2010.

${ }^{46}$ D. B. Nowak, ANSOM Project; see http://ansom.research.pdx.edu. 